YITP-11-51

\title{
Tensor models and hierarchy of n-ary algebras
}

\author{
Naoki SASAKURA* \\ Yukawa Institute for Theoretical Physics, Kyoto University, \\ Kyoto 606-8502, Japan
}

\begin{abstract}
Tensor models are generalization of matrix models, and are studied as models of quantum gravity. It is shown that the symmetry of the rank-three tensor models is generated by a hierarchy of n-ary algebras starting from the usual commutator, and the 3-ary algebra symmetry reported in the previous paper is just a single sector of the whole structure. The condition for the Leibnitz rules of the n-ary algebras is discussed from the perspective of the invariance of the underlying algebra under the n-ary transformations. It is shown that the n-ary transformations which keep the underlying algebraic structure invariant form closed finite n-ary Lie subalgebras. It is also shown that, in physical settings, the 3-ary transformation practically generates only local infinitesimal symmetry transformations, and the other more non-local infinitesimal symmetry transformations of the tensor models are generated by higher n-ary transformations.
\end{abstract}

*sasakura@yukawa.kyoto-u.ac.jp 


\section{Introduction}

Tensor models have originally been introduced [1, 2, 3] to describe the simplicial quantum gravity in general dimensions higher than two. The formulation has later been extended to describe spin foam and loop quantum gravities by considering Lie-group valued indices [4, 5, 6]. These models with group indices, so called group field theory, are actively studied with interesting recent progress [7]-[14].

The tensor models discussed in this paper are the simplest ones, which have a tensor with three indices as their only dynamical variable. By identifying the rank-three tensor as the structure constant of the algebra of functions on a fuzzy space, the rank-three tensor models can be regarded as theory of a dynamical fuzzy space [15, 16]. In the previous paper [16], it has been shown that the rank-three tensor models can be described algebraically and their symmetry transformations are generated by 3-ary transformations.

3-ary algebras [17, 18] have first been introduced in physics by Nambu [19], and have recently attracted much attention in the context of M-theory [20, 21, 22]. Higher n-ary algebras have also appeared in the description of various dimensional fuzzy spaces [23]. Since the rankthree tensor models are expected to describe fuzzy spaces corresponding to any dimensional continuous spaces [24]-[31], it would be natural that higher n-ary algebras also appear in the rank-three tensor models. The main purpose of this paper is to point out that, in fact, higher n-ary transformations also generate the symmetry of the rank-three tensor models.

This paper is organized as follows. In the following section, the correspondence between the rank-three tensor models and fuzzy spaces is recapitulated. The key property is the cyclic condition for the algebra of functions on a fuzzy space, which corresponds to the generalized hermiticity condition in the rank-three tensor models. In Section 3 , the general method is shown of constructing the n-ary algebras which generate the symmetry transformations of the rank-three tensor models. In Section 4, it is discussed how the Lie algebraic structure of the symmetry of the rank-three tensor models is incorporated in the hierarchical structure of the nary algebras. In Section 5, Leibnitz rules are shown to be satisfied by the n-ary transformations which generate the symmetry of the underlying algebra. Then they are shown to form closed finite n-ary Lie subalgebras. In Section 6, the roles of the higher n-ary algebras in the tensor models are discussed. Higher n-ary transformations are shown to correspond to more non-local infinitesimal symmetry transformations of the rank-three tensor models. The final section is devoted to summary and future prospects. 


\section{Tensor models and corresponding algebras}

In this section, I will briefly recapitulate the correspondence between the rank-three tensor models and fuzzy spaces, which has been presented in [16].

The tensor models [1, 2, 3] in this paper have a complex dynamical variable $M_{a b c}(a, b, c=$ $1,2, \ldots, N)$, which is a tensor with three indices and satisfies the generalized hermiticity condition,

$$
M_{a b c}=M_{b c a}=M_{c a b}=M_{b a c}^{*}=M_{a c b}^{*}=M_{c b a}^{*},
$$

where $*$ denotes complex conjugation. As degrees of freedom, this complex dynamical variable is equivalent to the following real dynamical variable defined by

$$
C_{a b c}=M_{a b c}+M_{b a c}+i\left(M_{a b c}-M_{b a c}\right)
$$

where $C_{a b c}$ satisfies the cyclicity condition,

$$
C_{a b c}=C_{b c a}=C_{c a b}
$$

A fuzzy space in this paper is characterized by the algebra of real functions $\left\{\phi_{a} \mid a=\right.$ $1,2, \ldots, N\}$ on it as

$$
\phi_{a} \phi_{b}=f_{a b}^{c} \phi_{c}
$$

where $f_{a b}^{c}$ is the real structure constant of the algebra. I also assume there exists a metric,

$$
\left\langle\phi_{a} \mid \phi_{b}\right\rangle=h_{a b}
$$

where $<\mid>$ is real, symmetric, $h_{a b}=h_{b a}$, and bilinear.

The correspondence between the rank-three tensor models and the fuzzy space is assumed to be given by

$$
C_{a b c}=f_{a b} c^{\prime} h_{c^{\prime} c}
$$

Then the cyclicity condition (3) of $C_{a b c}$ implies the following cyclicity condition for the algebra and the metric,

$$
\left\langle\phi_{a} \phi_{b} \mid \phi_{c}\right\rangle=\left\langle\phi_{b} \phi_{c} \mid \phi_{a}\right\rangle=\left\langle\phi_{c} \phi_{a} \mid \phi_{b}\right\rangle=\left\langle\phi_{a} \mid \phi_{b} \phi_{c}\right\rangle=\left\langle\phi_{b} \mid \phi_{c} \phi_{a}\right\rangle=\left\langle\phi_{c} \mid \phi_{a} \phi_{b}\right\rangle
$$

This is the key property for the following discussions. 


\section{General construction of metric invariant n-ary trans- formations}

In the previous paper [16], the infinitesimal linear transformations which keep the metric (50) invariant are shown to generate the symmetry of the rank-three tensor models, and a 3-ary transformation of this kind has been given explicitly. In this section, I will discuss the extension to n-ary transformations.

For illustration, let me start with reviewing the usual commutator from the viewpoint of this paper. By regarding the indices $b, c$ of $f_{a b} c$ as matrix indices, the algebra (4) can be regarded as a linear transformation expressed as

$$
\left(\phi_{a} ; \phi_{b}\right) \equiv \phi_{a} \phi_{b}=f_{a b}^{c} \phi_{c}
$$

where $\phi_{a}$ specifies the linear transformation, and $\phi_{b}$ is the objective of the linear transformation. From the property (17), one finds that

$$
\left\langle\phi_{b} \mid\left(\phi_{a} ; \phi_{c}\right)\right\rangle=\left\langle\phi_{b} \mid \phi_{a} \phi_{c}\right\rangle=\left\langle\phi_{b} \phi_{a} \mid \phi_{c}\right\rangle=\left\langle\overline{\left(\phi_{a} ; \phi_{b}\right)} \mid \phi_{c}\right\rangle
$$

where $\overline{(;)}$ denotes the transpose linear transformation given by

$$
\overline{\left(\phi_{a} ; \phi_{b}\right)} \equiv \phi_{b} \phi_{a}
$$

Here again $\phi_{a}$ specifies the linear transformation and $\phi_{b}$ is regarded as the objective of the transpose linear transformation. The transpose of a transpose is identical, because, due to $h_{a b}=h_{b a}$, the bra and ket states in (9) can be converted as

$$
\left\langle\left(\phi_{a} ; \phi_{c}\right) \mid \phi_{b}\right\rangle=\left\langle\phi_{c} \mid \overline{\left(\phi_{a} ; \phi_{b}\right)}\right\rangle \text {. }
$$

This is expressed as

$$
\overline{\overline{\left(\phi_{a} ; \phi_{b}\right)}}=\left(\phi_{a} ; \phi_{b}\right)
$$

Now let me define a linear transformation $[;]$ by

$$
\left[\phi_{a} ; \phi_{b}\right] \equiv\left(\phi_{a} ; \phi_{b}\right)-\overline{\left(\phi_{a} ; \phi_{b}\right)}
$$

Then one can show, from (9) and (11), the invariance of the metric,

$$
\left\langle\phi_{b} \mid\left[\phi_{a} ; \phi_{c}\right]\right\rangle=-\left\langle\left[\phi_{a} ; \phi_{b}\right] \mid \phi_{c}\right\rangle
$$

under the infinitesimal linear transformation $[;]$. In fact, this linear transformation is the usual commutator $\left[\phi_{a} ; \phi_{b}\right]=\phi_{a} \phi_{b}-\phi_{b} \phi_{a}$. 
The above procedure can be extended to products of any number of $\phi_{a}$ 's,

$$
\left(\phi_{a_{1}}, \phi_{a_{2}}, \ldots, \phi_{a_{p}}, s ; \phi_{b}\right)=\text { product of } \phi_{a_{1}}, \phi_{a_{2}}, \ldots, \phi_{a_{p}}, \phi_{b} \text { in way } s .
$$

Here $\phi_{a_{1}}, \phi_{a_{2}}, \ldots, \phi_{a_{p}}$ specify the linear transformation, $\phi_{b}$ is regarded as the objective of the linear transformation, and $s$ symbolically specifies the way of product. By moving $\phi_{a_{i}}$ 's from the ket state to the bra state with the use of the cyclic property (7), one can obtain the transpose linear transformation satisfying

$$
\left\langle\phi_{b} \mid\left(\phi_{a_{1}}, \phi_{a_{2}}, \ldots, \phi_{a_{p}}, s ; \phi_{c}\right)\right\rangle=\left\langle\left(\phi_{a_{1}}, \phi_{a_{2}}, \ldots, \phi_{a_{p}}, \bar{s} ; \phi_{b}\right) \mid \phi_{c}\right\rangle,
$$

where $\bar{s}$ symbolically denotes the transpose, namely,

$$
\left(\phi_{a_{1}}, \phi_{a_{2}}, \ldots, \phi_{a_{p}}, \bar{s} ; \phi_{b}\right) \equiv \overline{\left(\phi_{a_{1}}, \phi_{a_{2}}, \ldots, \phi_{a_{p}}, s ; \phi_{b}\right)} .
$$

As above, the transpose of a transpose is identical,

$$
\overline{\bar{s}}=s,
$$

which is a direct consequence of $h_{a b}=h_{b a}$.

Now let me define

$$
\left[\phi_{a_{1}}, \phi_{a_{2}}, \ldots, \phi_{a_{p}}, s ; \phi_{b}\right]=\left(\phi_{a_{1}}, \phi_{a_{2}}, \ldots, \phi_{a_{p}}, s ; \phi_{b}\right)-\left(\phi_{a_{1}}, \phi_{a_{2}}, \ldots, \phi_{a_{p}}, \bar{s} ; \phi_{b}\right) .
$$

From (16) and (18), one can show that the metric (5) is invariant under the linear transformation (19) as

$$
\left\langle\left[\phi_{a_{1}}, \phi_{a_{2}}, \ldots, \phi_{a_{p}}, s ; \phi_{b}\right] \mid \phi_{c}\right\rangle=-\left\langle\phi_{b} \mid\left[\phi_{a_{1}}, \phi_{a_{2}}, \ldots, \phi_{a_{p}}, s ; \phi_{c}\right]\right\rangle .
$$

From (18) and (19), it is obvious that

$$
\left[\phi_{a_{1}}, \phi_{a_{2}}, \ldots, \phi_{a_{p}}, s ; \phi_{b}\right]=-\left[\phi_{a_{1}}, \phi_{a_{2}}, \ldots, \phi_{a_{p}}, \bar{s} ; \phi_{b}\right] .
$$

The 3-ary transformation studied in the previous paper [16] is just a special case of the above general procedure. In the case, the starting linear transformation is taken to be

$$
\left(\phi_{a}, \phi_{b} ; \phi_{c}\right)=\left(\phi_{a} \phi_{c}\right) \phi_{b}
$$

By applying the above procedure, one obtains

$$
\begin{aligned}
\overline{\left(\phi_{a}, \phi_{b} ; \phi_{c}\right)} & =\left(\phi_{b} \phi_{c}\right) \phi_{a}, \\
{\left[\phi_{a}, \phi_{b} ; \phi_{c}\right] } & =\left(\phi_{a}, \phi_{b} ; \phi_{c}\right)-\overline{\left(\phi_{a}, \phi_{b} ; \phi_{c}\right)}=\left(\phi_{a} \phi_{c}\right) \phi_{b}-\left(\phi_{b} \phi_{c}\right) \phi_{a},
\end{aligned}
$$

which is indeed the 3-ary transformation studied in [16]. 


\section{Violation of Leibnitz rules and hierarchy of n-ary al- gebras}

Since the infinitesimal linear transformations which keep the metric $h_{a b}$ invariant form a Lie algebra, it would be interesting to discuss the Lie algebraic structure of the above n-ary transformations. Let me start with the following product of $(p+1)$ - and $(q+1)$-ary linear transformations,

$$
\left(\phi_{a_{1}}, \phi_{a_{2}}, \ldots, \phi_{a_{p+q}}, s * t ; \phi_{b}\right) \equiv\left[\phi_{a_{1}}, \phi_{a_{2}}, \ldots, \phi_{a_{p}}, s ;\left[\phi_{a_{p+1}}, \phi_{a_{p+2}}, \ldots, \phi_{a_{p+q}}, t ; \phi_{b}\right]\right] .
$$

In general, this product is not an infinitesimal transformation which keeps the metric $h_{a b}$ invariant. The transpose of the transformation is given by

$$
\left(\phi_{a_{1}}, \phi_{a_{2}}, \ldots, \phi_{a_{p+q}}, \overline{s * t} ; \phi_{b}\right)=\left[\phi_{a_{p+1}}, \phi_{a_{p+2}}, \ldots, \phi_{a_{p+q}}, t ;\left[\phi_{a_{1}}, \phi_{a_{2}}, \ldots, \phi_{a_{p}}, s ; \phi_{b}\right]\right] .
$$

This is because

$$
\begin{aligned}
\left\langle\phi_{b} \mid\left(\phi_{a_{1}}, \phi_{a_{2}}, \ldots, \phi_{a_{p+q}}, s * t ; \phi_{c}\right)\right\rangle & =\left\langle\phi_{b} \mid\left[\phi_{a_{1}}, \phi_{a_{2}}, \ldots, \phi_{a_{p}}, s ;\left[\phi_{a_{p+1}}, \phi_{a_{p+2}}, \ldots, \phi_{a_{p+q}}, t ; \phi_{c}\right]\right]\right\rangle \\
& =-\left\langle\left[\phi_{a_{1}}, \phi_{a_{2}}, \ldots, \phi_{a_{p}}, s ; \phi_{b}\right] \mid\left[\phi_{a_{p+1}}, \phi_{a_{p+2}}, \ldots, \phi_{a_{p+q}}, t ; \phi_{c}\right]\right\rangle \\
& =\left\langle\left[\phi_{a_{p+1}}, \phi_{a_{p+2}}, \ldots, \phi_{a_{p+q}}, t ;\left[\phi_{a_{1}}, \phi_{a_{2}}, \ldots, \phi_{a_{p}}, s ; \phi_{b}\right]\right] \mid \phi_{c}\right\rangle,
\end{aligned}
$$

where I have used (20). Therefore the commutator of the linear transformations, $s$ and $t$, has the form discussed in the previous section,

$$
\begin{aligned}
{\left[\phi_{a_{1}}, \ldots, \phi_{a_{p}}, s ;\left[\phi_{a_{p+1}}, \ldots, \phi_{a_{p+q}}, t ; \phi_{b}\right]\right]-\left[\phi_{a_{p+1}}, \ldots, \phi_{a_{p+q}}, t ;\left[\phi_{a_{1}}, \ldots, \phi_{a_{p}}, s ; \phi_{b}\right]\right] } \\
=\left(\phi_{a_{1}}, \phi_{a_{2}}, \ldots, \phi_{a_{p+q}}, s * t ; \phi_{b}\right)-\left(\phi_{a_{1}}, \phi_{a_{2}}, \ldots, \phi_{a_{p+q}}, \overline{s * t} ; \phi_{b}\right) .
\end{aligned}
$$

This shows that the commutator gives an infinitesimal linear transformation which keeps the metric invariant. This is of course expected, because the infinitesimal linear transformations which keep $h_{a b}$ invariant form a Lie algebra.

The exact form of the right-hand side of (27) depends on the structure of the algebra (4). If the n-ary transformations, $s$ and $t$, satisfy the Leibnitz rule (or so called fundamental identity) given by

$$
\begin{aligned}
{\left[\phi_{a_{1}}, \ldots, \phi_{a_{p}}, s ;\left[\phi_{a_{p+1}}, \ldots, \phi_{a_{p+q}}, t ; \phi_{b}\right]\right]=} & {\left[\left[\phi_{a_{1}}, \ldots, \phi_{a_{p}}, s ; \phi_{a_{p+1}}\right], \phi_{a_{p+2}}, \ldots, \phi_{a_{p+q}}, t ; \phi_{b}\right] } \\
& +\left[\phi_{a_{p+1}},\left[\phi_{a_{1}}, \ldots, \phi_{a_{p}}, s ; \phi_{a_{p+2}}\right], \ldots, \phi_{a_{p+q}}, t ; \phi_{b}\right] \\
& +\cdots
\end{aligned}
$$




$$
+\left[\phi_{a_{p+1}}, \ldots, \phi_{a_{p+q}}, t ;\left[\phi_{a_{1}}, \ldots, \phi_{a_{p}}, s ; \phi_{b}\right]\right]
$$

then the right-hand side of (27) can be expressed by $s$ or $t$ linear transformations, and the n-ary transformations will close by themselves. In general, however, the Leibnitz rules do not hold, and the right-hand side of (27) will be given by a linear summation of $(p+q+1)$-ary transformations,

$$
\sum_{s} c_{s}\left[\phi_{a_{1}}, \cdots, \phi_{a_{p+q}}, s ; \phi_{b}\right],
$$

where each [; ] has the form defined in (19), and $c_{s}$ are real coefficients. Thus to incorporate the Lie algebraic structure of the metric invariant n-ary transformations, it is in general necessary to consider the whole hierarchy of the n-ary transformations of the form (19)).

\section{Condition for Leibnitz rules}

In this section, I will discuss the condition for the Leibnitz rules to hold from the perspective of the invariance of the underlying algebra.

The $(p+1)$-ary linear transformation defined in (19) may be expressed as a tensorial form like

$$
\left[\phi_{a_{1}}, \phi_{a_{2}}, \ldots, \phi_{a_{p}}, s ; \phi_{b}\right]=M_{a_{1} \ldots a_{p} b}^{s}{ }^{c} \phi_{c},
$$

where $M_{a_{1} \ldots a_{p} b}^{s}{ }^{c}$ are real. With $M_{a_{1} \ldots a_{p} b}^{s}$, the invariance of the metric $h_{a b}$ under the infinitesimal linear transformation (30) can be expressed as

$$
\begin{aligned}
0 & =\left\langle\left[\phi_{a_{1}}, \phi_{a_{2}}, \ldots, \phi_{a_{p}}, s ; \phi_{b}\right] \mid \phi_{c}\right\rangle+\left\langle\phi_{b} \mid\left[\phi_{a_{1}}, \phi_{a_{2}}, \ldots, \phi_{a_{p}}, s ; \phi_{c}\right]\right\rangle \\
& =M_{a_{1} \ldots a_{p} b}^{s} h_{b^{\prime} c}+M_{a_{1} \ldots a_{p} c}^{s} h_{b c^{\prime}} .
\end{aligned}
$$

This implies the antisymmetry of the last two indices of $M$,

$$
M_{a_{1} \ldots a_{p} b c}^{s}=-M_{a_{1} \ldots a_{p} c b}^{s},
$$

where $M_{a_{1} \ldots a_{p} b c}^{s} \equiv M_{a_{1} \ldots a_{p} b}^{s}{ }^{d} h_{d c}$.

Now let me assume that the algebra (4) is invariant under the infinitesimal linear transformation (30) for a specific choice of $\phi_{a_{1}}, \phi_{a_{2}}, \ldots, \phi_{a_{p}}, s$. It is straightforward to extend the following discussions for linear combinations of $(p+1)$-ary linear transformations which keep (4) invariant. The assumption can be expressed by the following invariance of the structure constant of the algebra,

$$
M_{a_{1} \ldots a_{p} b}^{s} b_{b^{\prime} c}{ }^{d}+M_{a_{1} \ldots a_{p} c}^{s} c^{c^{\prime}} f_{b c^{\prime}}{ }^{d}-M_{a_{1} \ldots a_{p} d^{d^{\prime}}}^{s} f_{b c}{ }^{d^{\prime}}=0 .
$$


Or, in an algebraic form, this is equivalent to the Leibnitz rule,

$$
\left[\phi_{a_{1}}, \phi_{a_{2}}, \ldots, \phi_{a_{p}}, s ; \phi_{b} \phi_{c}\right]=\left[\phi_{a_{1}}, \phi_{a_{2}}, \ldots, \phi_{a_{p}}, s ; \phi_{b}\right] \phi_{c}+\phi_{b}\left[\phi_{a_{1}}, \phi_{a_{2}}, \ldots, \phi_{a_{p}}, s ; \phi_{c}\right]
$$

for any $\phi_{b}$ and $\phi_{c}$.

By iteratively applying (34), it is obvious that the objective of the linear transformation can be any product of any number of $\phi$ 's for the Leibnitz rules to hold. Therefore, the Leibnitz rules for the n-ary transformations, or so called fundamental identities, hold as

$$
\begin{aligned}
{\left[\phi_{a_{1}}, \ldots, \phi_{a_{p}}, s ;\left[\phi_{b_{1}}, \ldots, \phi_{b_{q}}, t ; \phi_{c}\right]\right]=} & {\left[\left[\phi_{a_{1}}, \ldots, \phi_{a_{p}}, s ; \phi_{b_{1}}\right], \ldots, \phi_{b_{q}}, t ; \phi_{c}\right] } \\
& +\left[\phi_{b_{1}},\left[\phi_{a_{1}}, \ldots, \phi_{a_{p}}, s ; \phi_{b_{2}}\right], \ldots, \phi_{b_{q}}, t ; \phi_{c}\right] \\
& +\cdots \\
& +\left[\phi_{b_{1}}, \ldots, \phi_{b_{q}}, t ;\left[\phi_{a_{1}}, \ldots, \phi_{a_{p}}, s ; \phi_{c}\right]\right] .
\end{aligned}
$$

Here it is important to note that $\phi_{b_{1}}, \ldots, \phi_{b_{q}}, t, \phi_{c}$ can be arbitrary, while $\phi_{a_{1}}, \ldots, \phi_{a_{p}}, s$ must be taken so that the linear transformation (30) be the symmetry of the algebra and satisfy (33) or (34).

Interesting structures appear when both $\left[\phi_{a_{1}}, \phi_{a_{2}}, \ldots, \phi_{a_{p}}, s ;\right]$ and $\left[\phi_{b_{1}}, \ldots, \phi_{b_{q}}, t ;\right]$ are taken to be the infinitesimal linear transformations being the symmetry of the underlying algebra. Then, as discussed in the previous section, due to the Leibnitz rule, the commutator of these linear transformations will not generate other n-ary linear transformations. Since the infinitesimal linear transformations which generate the symmetry of the underlying algebra form a Lie algebra, the n-ary linear transformation obtained from the commutator is again a symmetry transformation. This implies that the n-ary transformations which generate the symmetry of the underlying algebra will form closed finite n-ary Lie subalgebras.

\section{The rank-three tensor models and n-ary algebras}

The symmetry of the rank-three tensor models is the orthogonal group symmetry,

$$
C_{a b c} \rightarrow O_{a}{ }^{a^{\prime}} O_{b}{ }^{b^{\prime}} O_{c}{ }^{c^{\prime}} C_{a^{\prime} b^{\prime} c^{\prime}}, \quad O \in O(N, R)
$$

As discussed in [16], this orthogonal group symmetry of the tensor models can be identified with the symmetry of fuzzy spaces by partially gauge-fixing the latter symmetry with the gauge fixing condition,

$$
h_{a b}=\delta_{a b} .
$$


Then indeed the n-ary linear transformations (19) become the infinitesimal orthogonal group transformations, which keep $h_{a b}=\delta_{a b}$ invariant, and can be identified with the generators of the symmetry transformations (36) of the rank-three tensor models.

In the previous paper [16], it is argued that the 3-ary transformations,

$$
\delta_{b c} \phi_{a}=\left[\phi_{b}, \phi_{c} ; \phi_{a}\right]
$$

with [ ; ] defined in (23), will generate the symmetry transformations of the tensor models by taking various choices of the functions $\phi_{b}$ and $\phi_{c}$. Since the number of the independent elements of the Lie algebra $s o(N)$ agrees with the number of independent choices of $\phi_{b}$ and $\phi_{c}$ on account of the antisymmetry of the first two entries of the 3-ary transformation, the 3-ary transformations (38) will generate all the infinitesimal symmetry transformations of the tensor models, unless $C_{a b c}$ is fine-tuned not to be so.

The above statement is mathematically true, but this is not physically realistic. To explain this, for illustration, let me consider a class of $C_{a b c}$ with the following Gaussian forms,

$$
C_{x_{1} x_{2} x_{3}}=\exp \left[-\beta\left(\left(x_{1}-x_{2}\right)^{2}+\left(x_{2}-x_{3}\right)^{2}+\left(x_{3}-x_{1}\right)^{2}\right)\right],
$$

where $\beta$ is a positive real parameter with dimension (length) $)^{-2}$, the indices $x_{i}$ 's are $D$ dimensional coordinates, and

$$
x^{2} \equiv g_{\mu \nu} x^{\mu} x^{\nu},
$$

with a real symmetric two-tensor $g_{\mu \nu}$. The $g_{\mu \nu}$ is assumed to be a positive definite matrix. Physically the algebra*

$$
\phi_{x_{1}} \phi_{x_{2}}=C_{x_{1} x_{2} x_{3}} \phi_{x_{3}}
$$

which is obtained from (39) through (6) and (4) with (37), can be regarded as the algebra of the functions on a $D$-dimensional fuzzy flat space with fuzzy scale $1 / \sqrt{\beta}$ [32]. Here the function $\phi_{x}$ may be regarded as a fuzzy analogue of the delta function $\delta^{D}(z-x)$ on a usual continuous space.

With $\phi_{x}$, the 3-ary transformations are given by

$$
\delta_{x_{2} x_{3}} \phi_{x_{1}}=\left[\phi_{x_{2}}, \phi_{x_{3}} ; \phi_{x_{1}}\right] .
$$

Indeed one can numerically $\dagger^{-}$check that the 3-ary transformations (42) generate all the infinitesimal symmetry transformations of the tensor models by taking all the choices of $\phi_{x_{2}}$ and $\phi_{x_{3}}$. However, it is obvious, from the Gaussian damping form of (39), that, for the 3-ary

*The repeated index is assumed to be integrated over.

${ }^{\dagger}$ The continuous $x_{i}$ 's are discretized for the numerical check, and only the case $D=1$ is checked. 
transformations (42) to be significant, $x_{2}$ and $x_{3}$ must be taken within the range of order $1 / \sqrt{\beta}$ around $x_{1}$. Thus, practically, the 3 -ary transformations (42) only generate local (with fuzziness $1 / \sqrt{\beta}$ ) infinitesimal transformations, while the Lie generators for (36) of the tensor models contain non-local ones $o_{x_{1}}{ }^{x_{2}} \in s o(N)$ with arbitrary choices of $x_{1}$ and $x_{2}$.

The above conclusion does not depend on the choice of a basis of functions on a fuzzy space, nor is it restricted to the specific Gaussian form (39). Any physically acceptable fuzzy space must respect locality (with certain fuzziness), and a basis of functions may be taken so that each function be localized within a characteristic scale of fuzziness. Then products of two functions become significant only when the two functions are overlapping within the characteristic scale. Thus, practically, for general fuzzy spaces respecting locality, the 3-ary transformations can only generate local infinitesimal transformations, which are far less than all the infinitesimal symmetry transformations of the tensor models.

The other more non-local infinitesimal symmetry transformations will be obtained by considering higher n-ary transformations defined in (19). A $p$-ary transformation contains $(p-1)$ products of functions, and can be made significant by ordering the localized basis functions in a chain so that neighboring functions are overlapping within the characteristic scale of fuzziness. Since such a chain can be made arbitrarily long by considering an arbitrary number of functions, arbitrarily non-local infinitesimal symmetry transformations will be obtained by considering arbitrarily higher n-ary transformations. The necessity of higher n-ary transformations is also natural from the Lie algebraic structure of the symmetry of the tensor models, as discussed in Section 4, because successive applications of the local infinitesimal transformations will generate non-local transformations.

\section{Summary and future prospects}

In this paper, I have discussed the n-ary algebras for describing the symmetry of the tensor models. I have presented the general method to construct the n-ary transformations which generate the symmetry of the tensor models. The 3-ary algebra reported in the previous paper is just a particular case of the general method. The Lie algebraic structure of the symmetry of the tensor models can be incorporated by the hierarchical structure of the n-ary algebras. If there exist n-ary transformations which generate the symmetry of the underlying algebra, the Leibnitz rules hold for them, and they form closed finite n-ary Lie subalgebras. The 3-ary transformations can generally generate all the infinitesimal symmetry transformations of the tensor models, but in physical settings, they practically generate only local infinitesimal symmetry transformations due to the locality which should be respected by physically acceptable 
fuzzy spaces. The other more non-local infinitesimal symmetry transformations of the tensor models can be generated by higher n-ary transformations.

The starting key assumption of this paper is the cyclic property concerning the algebra of functions on a fuzzy space and its metric. This property allows systematic construction of the symmetry generators. The property has been derived in the context of the tensor models, but it is also interesting to set this assumption in the general context of fuzzy spaces. The notion of fuzzy space is an interesting candidate to replace the classical spacetime notion of general relativity. However, this new notion is currently lacking a general basic framework and is yet to be developed. The settings assumed for fuzzy spaces in this paper may hopefully shed new light in this direction.

\section{References}

[1] J. Ambjorn, B. Durhuus and T. Jonsson, "Three-Dimensional Simplicial Quantum Gravity And Generalized Matrix Models," Mod. Phys. Lett. A 6, 1133 (1991).

[2] N. Sasakura, "Tensor Model For Gravity And Orientability Of Manifold," Mod. Phys. Lett. A 6, 2613 (1991).

[3] N. Godfrey and M. Gross, "Simplicial Quantum Gravity In More Than Two-Dimensions," Phys. Rev. D 43, 1749 (1991).

[4] D. V. Boulatov, "A Model of three-dimensional lattice gravity," Mod. Phys. Lett. A 7 , 1629 (1992) arXiv:hep-th/9202074.

[5] H. Ooguri, "Topological lattice models in four-dimensions," Mod. Phys. Lett. A 7, 2799 (1992) arXiv:hep-th/9205090.

[6] R. De Pietri, L. Freidel, K. Krasnov and C. Rovelli, "Barrett-Crane model from a Boulatov-Ooguri field theory over a homogeneous space," Nucl. Phys. B 574, 785 (2000) arXiv:hep-th/9907154.

[7] S. Carrozza and D. Oriti, "Bounding bubbles: the vertex representation of 3d Group Field Theory and the suppression of pseudo-manifolds," arXiv:1104.5158 [hep-th].

[8] R. Gurau, "The complete 1/N expansion of colored tensor models in arbitrary dimension," arXiv:1102.5759 [gr-qc]]. 
[9] A. Baratin, F. Girelli and D. Oriti, "Diffeomorphisms in group field theories," Phys. Rev. D 83, 104051 (2011) arXiv:1101.0590 [hep-th]].

[10] R. Gurau, "The 1/N expansion of colored tensor models," arXiv:1011.2726 [gr-qc]].

[11] J. B. Geloun, R. Gurau, V. Rivasseau, "EPRL/FK Group Field Theory," Europhys. Lett. 92, 60008 (2010). arXiv:1008.0354 [hep-th]].

[12] R. Gurau, "Lost in Translation: Topological Singularities in Group Field Theory," Class. Quant. Grav. 27, 235023 (2010). arXiv:1006.0714 [hep-th]].

[13] R. Gurau, "Topological Graph Polynomials in Colored Group Field Theory," Annales Henri Poincare 11, 565-584 (2010). arXiv:0911.1945 [hep-th]].

[14] R. Gurau, "Colored Group Field Theory," Commun. Math. Phys. 304, 69 (2011) arXiv:0907.2582 [hep-th]].

[15] N. Sasakura, "An Invariant approach to dynamical fuzzy spaces with a three-index variable," Mod. Phys. Lett. A21, 1017-1028 (2006). hep-th/0506192.

[16] N. Sasakura, "Tensor models and 3-ary algebras," arXiv:1104.1463 [hep-th].

[17] J. M. Figueroa-O'Farrill, "Three lectures on 3-algebras," arXiv:0812.2865 [hep-th]].

[18] J. A. de Azcarraga and J. M. Izquierdo, "Topics on n-ary algebras," J. Phys. Conf. Ser. 284, 012019 (2011) arXiv:1102.4194 [math-ph]].

[19] Y. Nambu, "Generalized Hamiltonian dynamics," Phys. Rev. D7, 2405-2414 (1973).

[20] J. Bagger, N. Lambert, "Modeling Multiple M2's," Phys. Rev. D75, 045020 (2007). hep-th/0611108.

[21] A. Gustavsson, "Algebraic structures on parallel M2-branes," Nucl. Phys. B811, 66-76 (2009). [arXiv:0709.1260 [hep-th]].

[22] J. Bagger, N. Lambert, "Gauge symmetry and supersymmetry of multiple M2-branes," Phys. Rev. D77, 065008 (2008). arXiv:0711.0955 [hep-th]].

[23] J. DeBellis, C. Saemann and R. J. Szabo, "Quantized Nambu-Poisson Manifolds and n-Lie Algebras," J. Math. Phys. 51, 122303 (2010) [arXiv:1001.3275 [hep-th]].

[24] N. Sasakura, "An Invariant approach to dynamical fuzzy spaces with a three-index variable: Euclidean models," hep-th/0511154. 
[25] N. Sasakura, "Tensor model and dynamical generation of commutative nonassociative fuzzy spaces," Class. Quant. Grav. 23, 5397-5416 (2006). hep-th/0606066.

[26] N. Sasakura, "The Fluctuation spectra around a Gaussian classical solution of a tensor model and the general relativity," Int. J. Mod. Phys. A23, 693-718 (2008). arXiv:0706.1618 [hep-th]].

[27] N. Sasakura, "The Lowest modes around Gaussian solutions of tensor models and the general relativity," Int. J. Mod. Phys. A23, 3863-3890 (2008). arXiv:0710.0696 [hep-th]].

[28] N. Sasakura, "Emergent general relativity on fuzzy spaces from tensor models," Prog. Theor. Phys. 119, 1029-1040 (2008). [arXiv:0803.1717] [gr-qc]].

[29] N. Sasakura, "Gauge fixing in the tensor model and emergence of local gauge symmetries," Prog. Theor. Phys. 122, 309-322 (2009). [arXiv:0904.0046 [hep-th]].

[30] N. Sasakura, "Emergent general relativity in the tensor models possessing Gaussian classical solutions," AIP Conf. Proc. 1243, 76-86 (2010). [arXiv:0911.1170 [hep-th]].

[31] N. Sasakura, "A Renormalization procedure for tensor models and scalar-tensor theories of gravity," Int. J. Mod. Phys. A25, 4475-4492 (2010). arXiv:1005.3088 [hep-th]].

[32] Y. Sasai, N. Sasakura, "One-loop unitarity of scalar field theories on Poincare invariant commutative nonassociative spacetimes," JHEP 0609, 046 (2006). hep-th/0604194. 